\title{
Review on near-infrared heptamethine cyanine dyes as theranostic agents for tumor imaging, targeting, and photodynamic therapy
}

Changhong Shi Jason Boyang Wu Dongfeng Pan 


\title{
Review on near-infrared heptamethine cyanine dyes as theranostic agents for tumor imaging, targeting, and photodynamic therapy
}

\author{
Changhong Shi, ${ }^{a, *}$ Jason Boyang $\mathrm{Wu}^{\mathrm{b}}$ and Dongfeng Pan ${ }^{\mathrm{c}}$ \\ ${ }^{a} F$ ourth Military Medical University, Laboratory Animal Center, 169 West Changle Road, Xi'an, Shaanxi 710032, China \\ bSamuel Oschin Comprehensive Cancer Institute, Cedars-Sinai Medical Center, Uro-Oncology Research Program, \\ Department of Medicine, Los Angeles, California 90048, United States \\ 'University of Virginia, Department of Radiology, Charlottesville, Virginia 22908, United States
}

\begin{abstract}
A class of near-infrared fluorescence (NIRF) heptamethine cyanine dyes that are taken up and accumulated specifically in cancer cells without chemical conjugation have recently emerged as promising tools for tumor imaging and targeting. In addition to their fluorescence and nuclear imagingbased tumor-imaging properties, these dyes can be developed as drug carriers to safely deliver chemotherapy drugs to tumors. They can also be used as effective agents for photodynamic therapy with remarkable tumoricidal activity via photodependent cytotoxic activity. The preferential uptake of dyes into cancer but not normal cells is co-operatively mediated by the prevailing activation of a group of organic aniontransporting polypeptides on cancer cell membranes, as well as tumor hypoxia and increased mitochondrial membrane potential in cancer cells. Such mechanistic explorations have greatly advanced the current application and future development of NIRF dyes and their derivatives as anticancer theranostic agents. This review summarizes current knowledge and emerging advances in NIRF dyes, including molecular characterization, photophysical properties, multimodal development and uptake mechanisms, and their growing potential for preclinical and clinical use. @ 2016 Society of Photo-Optical Instrumentation Engineers (SPIE) [DOI: 10.1117/1.JBO.21.5 .050901]
\end{abstract}

Keywords: near-infrared fluorescence; heptamethine cyanine dyes; tumor imaging; targeting; photodynamic therapy. Paper 160020VR received Jan. 11, 2016; accepted for publication Apr. 20, 2016; published online May 10, 2016.

\section{Introduction}

Near-infrared fluorescence (NIRF) imaging agents have high extinction coefficients, large Stokes' shifts, and the ability to generate strong fluorescence emission at the range of 700 to $1000 \mathrm{~nm},{ }^{1}$ offering the possibility of in vivo cancer diagnosis. Their considerable advantages for in vivo imaging include stronger ligand labeling, signal strength and tissue absorbance, a wider range of imaging materials for coupling, and less background fluorescence. ${ }^{2-4}$ Several NIRF dyes have become commercially available, such as Cy5. $5^{5}$ and IRDye $800-\mathrm{CW},{ }^{6}$ which have been coupled with peptides or antibodies and successfully used for targeted visualization of neoplastic cancers in animal models. Indocyanine green (ICG), the only NIRF agent approved by the FDA in the United States for medical diagnostic application, has been widely used to differentiate benign from the malignant diseases in the clinic. ${ }^{7}$ However, most of these dyes display poor stability, rapid decomposition in polar solution, low quantum yield, and a lack of tumor-targeting specificity. $^{8}$

To be used for cancer imaging, conventional NIRF dyes require chemical conjugation of cancer-specific targeting ligands, such as metabolic substrates, ${ }^{9}$ cell-surface peptides, ${ }^{10}$ growth factors, ${ }^{11}$ antibodies, ${ }^{12}$ and cancer-specific cell-surface

*Address all correspondence to: Changhong Shi, E-mail: changhong@fmmu. edu.cn biomarkers. ${ }^{13}$ Thus, only limited specific types of cancers can be detected by these imaging probes due to tumor cell heterogeneity. Additionally, the specific imaging or targeting properties of tumor-targeted ligands may be altered by chemical conjugation. $^{14}$

Recently, a group of NIRF heptamethine cyanine dyes have emerged as more promising tools for cancer imaging and targeted therapy. These agents are heterocyclic polymethine cyanines with dual imaging and targeting properties, including IR-780, ${ }^{5}$ IR-783, ${ }^{6}$ IR-808, ${ }^{15}$ and MHI-148. ${ }^{16}$ They have demonstrated preferential accumulation and retention indifferent types of cancer cells (Table 1), with increased uptake in the mitochondria and lysosomes, but no accumulation in normal cells, enabling cancer-specific targeting without the need for chemical conjugation. For instance, after the NIRF dye IR-783 was administered into tumor-bearing mice $(0.375 \mathrm{mg} / \mathrm{kg}$, i.p. route), the fluorescence signals became detectable in select mouse organs (e.g., liver, kidney, lung, and heart) at $6 \mathrm{~h}$ and further showed preferential accumulation in tumor tissues at $24 \mathrm{~h}$ with minimal background autofluorescence retained in other organs. After $80 \mathrm{~h}$ since injection, the dye was cleared from all vital organs. They show low autofluorescence, but when combined with biological molecules, they induce strong fluorescence after being chemically modified to improve the stability of the molecules. ${ }^{19}$ Importantly, these dyes do not cause cytotoxicity or systemic toxicity in mice when administered in a dose range appropriate for imaging. ${ }^{6}$ 
Table 1 Summary of NIRF dye studies in different tumor cell lines and xenograft models.

\begin{tabular}{|c|c|c|c|}
\hline Tumor type & Tumor cell line & $\begin{array}{l}\text { Heptamethine } \\
\text { cyanine dye }\end{array}$ & References \\
\hline Human lung cancer & A549, NClH-460 & IR-808 & 17,18 \\
\hline $\begin{array}{l}\text { Human breast } \\
\text { cancer }\end{array}$ & $\begin{array}{l}\text { MCF-7, } \\
\text { MDA231 }\end{array}$ & & 17 \\
\hline Human hepatoma & $\begin{array}{l}\text { SMMC-7721, } \\
\text { HepG2 }\end{array}$ & & 17 \\
\hline $\begin{array}{l}\text { Human cervical } \\
\text { cancer }\end{array}$ & HeLa & & 17 \\
\hline $\begin{array}{l}\text { Human prostate } \\
\text { cancer }\end{array}$ & $\begin{array}{l}\text { PC-3, LNCap, } \\
\text { C42 }\end{array}$ & MHI-148 & $19-21$ \\
\hline Human leukemia & K562 & & 19,20 \\
\hline Dog breast cancer & $\begin{array}{l}\text { CHMp-5b, } \\
\text { CHMp-13a }\end{array}$ & & 22 \\
\hline $\begin{array}{l}\text { Dog prostate } \\
\text { cancer }\end{array}$ & ACE1 & & 22 \\
\hline \multirow[t]{2}{*}{$\begin{array}{l}\text { Human prostate } \\
\text { cancer }\end{array}$} & $\begin{array}{l}\text { PC-3, LNCap, } \\
\text { DU-145 }\end{array}$ & IR-783 & $6,14,19,23$ \\
\hline & $\begin{array}{c}\text { C4-2, } \\
\text { ARCaP }_{M}\end{array}$ & & 19 \\
\hline $\begin{array}{l}\text { Human breast } \\
\text { cancer }\end{array}$ & MCF-7 & & $14,19,24$ \\
\hline $\begin{array}{l}\text { Human cervical } \\
\text { cancer }\end{array}$ & HeLa & & 19 \\
\hline Human lung cancer & H358 & & 19 \\
\hline Human hepatoma & HepG2 & & 19 \\
\hline $\begin{array}{l}\text { Human pancreatic } \\
\text { cancer }\end{array}$ & MIA, PaCa-2 & & 19 \\
\hline Human renal cancer & $\begin{array}{l}\text { SN12C, } \\
\text { ACHN, } \\
\text { Caki-1 }\end{array}$ & & 16 \\
\hline Human leukemia & K562 & & 19 \\
\hline Human glioblastoma & $\begin{array}{l}\text { U87, U251, } \\
\text { T98T }\end{array}$ & & 25 \\
\hline $\begin{array}{l}\text { Human breast } \\
\text { cancer }\end{array}$ & $\begin{array}{l}\text { MCF-7, } \\
\text { MDA231 }\end{array}$ & $\begin{array}{l}\text { IR-780 } \\
\text { lodide }\end{array}$ & $14,26,27$ \\
\hline $\begin{array}{l}\text { Human cervical } \\
\text { cancer }\end{array}$ & HeLa & & 27 \\
\hline $\begin{array}{l}\text { Human } \\
\text { osteosarcoma }\end{array}$ & MG-63 & & 27 \\
\hline Human lung cancer & A549, A549/DR & & 28,29 \\
\hline Human hepatoma & HepG2 & & 28 \\
\hline
\end{tabular}

Table 1 (Continued).

\begin{tabular}{|c|c|c|c|}
\hline Tumor type & Tumor cell line & $\begin{array}{l}\text { Heptamethine } \\
\text { cyanine dye }\end{array}$ & References \\
\hline Human glioma & U251, GL261 & & 28 \\
\hline $\begin{array}{l}\text { Human renal } \\
\text { cancer }\end{array}$ & $\begin{array}{l}\text { SN12C, } \\
\text { ACHN, } \\
\text { Caki-1 }\end{array}$ & & 16 \\
\hline $\begin{array}{l}\text { Human prostate } \\
\text { cancer }\end{array}$ & PC-3, LNCap & & 5 \\
\hline $\begin{array}{l}\text { Human breast } \\
\text { tumor }\end{array}$ & MCF-7 & $\begin{array}{l}\text { PC-1001, } \\
\text { PC-1007 }\end{array}$ & 30-32 \\
\hline $\begin{array}{l}\text { Dog testicular } \\
\text { tumors }\end{array}$ & $\begin{array}{c}\text { Spontaneous } \\
\text { tumor }\end{array}$ & & 22 \\
\hline
\end{tabular}

\section{Physical and Chemical Characteristics of Near-Infrared Fluorescence Heptamethine Cyanine Dyes}

The photophysical properties of heptamethine cyanine dye to that of ICG are summarized in Table 2. The singlet oxygen $\left({ }^{1} \mathrm{O}_{2}\right)$ yields of IR-783 and ICG are 0.007 and 0.008 , respectively. Although IR-783 is not excitable at longer NIR wavelengths compared with ICG, IR-783 could be the best candidate for conjugation to photosensitizer by owning higher absorptivity $\left(\varepsilon=261,000 \mathrm{M}^{-1} \mathrm{~cm}^{-1}\right)$ and fluorescence efficiency $\left(\Phi_{\mathrm{Fl}}=0.084\right) .{ }^{36}$ NIRF dyes share a common resonance structure consisting of a polymethyl group and a nitrogen atom, which are involved in the formation of a conjugated heterocycle at both sides. Traditional cyanine dyes display poor photostability, self-aggregation, and low quantum-yield solution. ${ }^{37}$ Previous studies showed that both the excitation and emission wavelengths of these compounds were increased by the extension of the polymethyl chain. Most cyanine dyes are prone to self-aggregation, which is affected by the length of the carbon chain. ${ }^{15}$ Moreover, the fluorescence quantum-yield is decreased in aqueous solution. ${ }^{26}$ To solve these problems, a variety of structural modifications have been attempted in these dyes. ${ }^{38}$ Acyclohexenyl ring has been introduced into the central polymethyl acetylene chain, leading to significantly increased photostability and fluorescence intensity. ${ }^{39}$ The introduction of asulfate group not only avoided the weakening of fluorescence signals from nonspecific hydrophobic exchange of the fluorescent group in aqueous solution with other molecules but also reduced the self-aggregation of these dyes. ${ }^{40}$ The polymethyl

Table 2 Photophysical properties of NIRF dyes.

\begin{tabular}{lccccc}
\hline & & & & \\
Dye & MW & $\begin{array}{c}\lambda_{\max . A b s} \\
(\mathrm{~nm})\end{array}$ & $\begin{array}{c}\lambda_{\max . e m} \\
(\mathrm{~nm})\end{array}$ & $\begin{array}{c}\text { Fluorescence } \\
\text { quantum yields }\end{array}$ & References \\
\hline IR-783 & 750 & 766 & 782 & 0.186 (PBS) & 33 \\
MHI-148 & 705 & 785 & 808 & 0.12 (Ethanol) & 1 \\
IR-780 & 667 & 777 & 823 & 0.17 (Ethanol) & 34 \\
ICG & 775 & 780 & 812 & 0.012 (PBS) & 35 \\
\hline
\end{tabular}


acetylene chain in the structure inhibits the internal energy conversion and polymerization, which increases the intensity and stability of the fluorescent signals. ${ }^{19,41}$ However, a longer polymethyl chain in the middle structure is often associated with lower structural stability, which can be improved by introducing ring groups to increase the rigidity of the structure by reducing internal energy conversion and polymerization. ${ }^{40}$

Several representative heptamethine cyanine dyes have been extensively studied for their common chemical structures (Fig. 1). The carboxyl side chain of MHI-148 can be conjugated with chemotherapy drugs or isotopes, showing chemical operability and slow removal in vivo. ${ }^{19}$ By contrast, the alkyl side chain of IR-780 is difficult to modify. ${ }^{42}$ Both MHI-148 and IR-780 are lipid-soluble compounds and show toxic effects, which are tolerated in mice but limit the application of these dyes in vivo. The sulfonic acid group in the side chain of IR783 allows better water solubility and lower toxicity through a quick clearance in vivo, but its structural modification remains difficult. ${ }^{19}$ With the indolenine nucleus, a chlorinated cyclohexenyl center and a sulfonate group, IR-783 showed the best selectivity for tumor imaging. ${ }^{38}$ The development of novel dyes with improved chemical properties that maintain cancer-targeting specificity but also possess dual water- and lipid-soluble characteristics with appropriate removal efficiency and low toxicity in vivo is urgently needed. Such new dyes are also expected to facilitate chemical modifications, such as conjugations with labeling probes, radionuclides and chemotherapy drugs, to improve the sensitivity of recognition by cancer cells. In line with these considerations, we have designed and synthesized a novel NIRF dye, MHI-148-783, whose chemical structure is shown in Fig. 1. MHI-148-783 has the sulfonic acid group of IR-783 in one side chain, with MHI-148's carboxyl group in the other side chain.

Conventional organic NIR dyes are poor in photobleaching due to a chemical reaction with reactive oxygen species (ROS), such as ${ }^{1} \mathrm{O}_{2}$ production. ${ }^{43}$ It was reported that NIR dye croconaine (Croc) efficiently converts 808 -nm laser light into heat without the production of ${ }^{1} \mathrm{O}_{2}$ and thus exhibits attractive photothemal properties. ${ }^{44}$ These properties allow Croc-doped lipidpolymer hybrid nanoparticles to absorb strongly at $808 \mathrm{~nm}$ and generate clean laser-induced heating without producing ${ }^{1} \mathrm{O}_{2}$ to become resistant to photobleaching effectively. ${ }^{45}$

\section{Near-Infrared Fluorescence Dye-Mediated Tumor Imaging and Targeting}

Conventional NIRF polymethine cyanine organic dyes, such as ICG, lack tumor-targeting capability. Approaches utilizing NIRF dyes in cancer imaging require chemical conjugation to achieve active targeting and to improve delivery efficacy. ${ }^{1}$ Very few organic NIRF dyes in their native forms possess the ability to target tumors with high specificity. ${ }^{16}$ Recently, a unique group of NIRF heptamethine cyanine dyes have been demonstrated for specific transport into tumor cells by distinguishing malignant from normal/benign cells in xenograft or spontaneous tumor models in vivo as well as surgically resected tumor specimens ex vivo.

\subsection{Tumor Cell and Xenograft Models}

These organic dyes have been reported to be directly taken up and preferentially accumulate in the mitochondria of tumor without inference in normal tissues in preclinical models, including mice and dogs. ${ }^{5,6,15,16,20,22}$ For example, Pz 247, a chiral porphyazine NIRF agent without chemical conjugation, show tumor-targeting property and exhibited preferential retention in the tumor cells of human breast tumor xenografts subcutaneously implanted in mice. ${ }^{46}$ Heptamethine cyanine dyebased NIRF imaging is becoming an attractive modality for cancer detection with the acquisition of real-time pathophysiologic information. At the cellular level, these NIRF agents also show preferential uptake in cancer cells but not normal cells, as demonstrated in many different types of cancer cells including cultured, circulating and disseminated tumor cells. ${ }^{5,6,16}$

\subsection{Patient-Derived Tumor Xenograft Models}

In addition to solid tumors, these NIRF dyes can detect tumor cells either in the interstitial fluid or in the form of circulating tumor cells with high sensitivity and specificity. ${ }^{19}$ The analysis of malignant tumor cells in splanchnocoele fluid by NIRF imaging has become a fast and reliable method to monitor disease

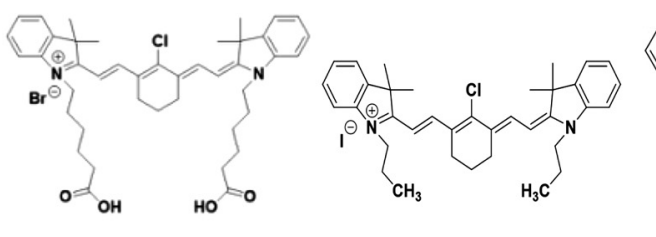

$\mathrm{MHI}-148$

Pz 247

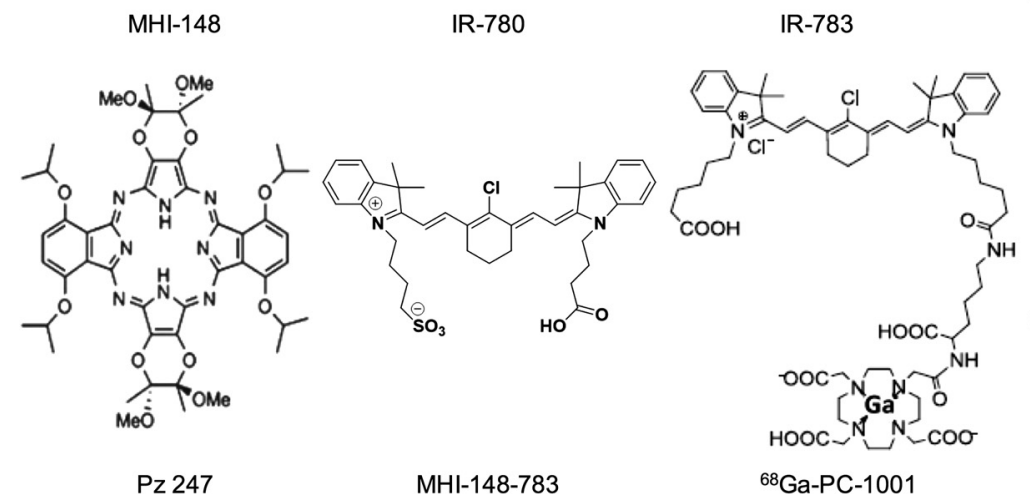

IR-780

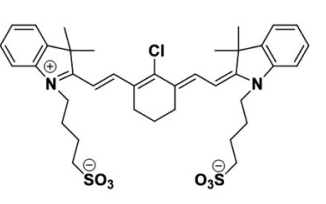

IR-783
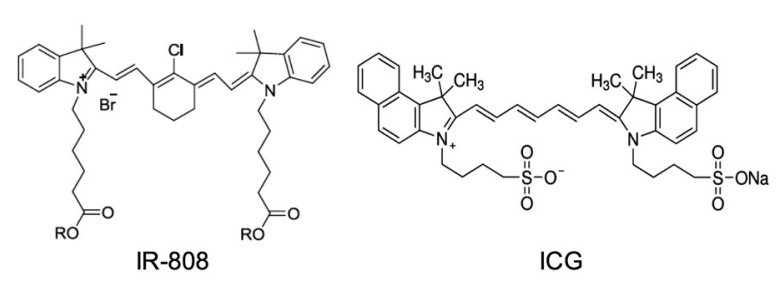

ICG
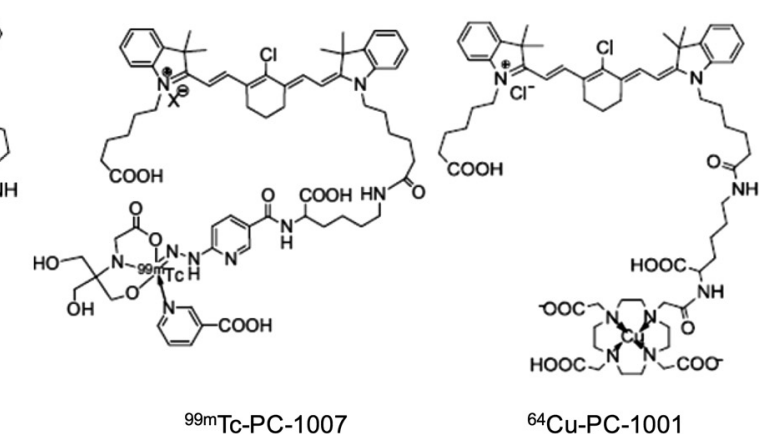

Fig. 1 Chemical structures of NIRF heptamethine cyanine dyes as dual imaging and targeting agents. 
progression in cancer patients. ${ }^{6,47}$ In recent years, patientderived tumor xenograft (PDX) models established by transplanting human fresh tumor tissues into immunodeficient mice have become another valuable tool for maintaining intact tumor profiles, such as tumor heterogeneity. They can be used to screen and test anticancer drug efficacy tailored for individualized treatment. ${ }^{48}$ Conventional PDX models are visualized in vivo by luminescence-based optical imaging after labeling tumors with a luciferase reporter gene, which is time-consuming and complicated. ${ }^{49}$ On the other hand, the alternative in vitro screening of PDX-derived tumor cells often results in a loss of tumor cell subsets and information on tumor heterogeneity. ${ }^{50}$ With the introduction of heptamethine cyanine dyes, we were able to characterize the PDX models by NIRF imaging by readily capturing subrenally grafted deep tissues. A wide spectrum of PDX models harboring different types of tumor samples, including gastric cancer, liver cancer, bladder cancer and renal cancer, were identified by NIRF imaging by the exclusive exhibition of fluorescence signals in the renal area of mice (Fig. 2), which was further confirmed concurrently by tumor histology using H\&E stain (data not shown).

\subsection{Clinical Experience}

NIRF dyes show tumor-specific targeting inhuman tumor samples. ${ }^{30}$ The fact that these dyes possess dual tumor-imaging and targeting capabilities suggests possible applications for the real-time monitoring of tumor growth as well as the recognition of surgical margins during surgery, which would significantly advance the accurate localization of tumor margins and thus improve surgical effects by reducing the possibility of missing positive lesions. ${ }^{51}$ In agreement with this idea, recent evidence has demonstrated that surgical resection of hepatic metastases with the assistance of NIRF imaging accurately recognized benign and malignant lesions of the liver and, more importantly, facilitated tumor detection with a focus of less than $5 \mathrm{~mm}^{52}$

In another preclinical trial, MHI-148 dye was applied for direct imaging of clinical tumor specimens, where the entire kidney removed surgically from a renal cell carcinoma (RCC) patient was subjected to immediate perfusion with saline-dissolved MHI-148 dye followed by ex vivo NIRF imaging. In this first-in-man ex vivo test without a tumor host, the NIRF signals were clearly detected in tumor areas in sharp contrast to the

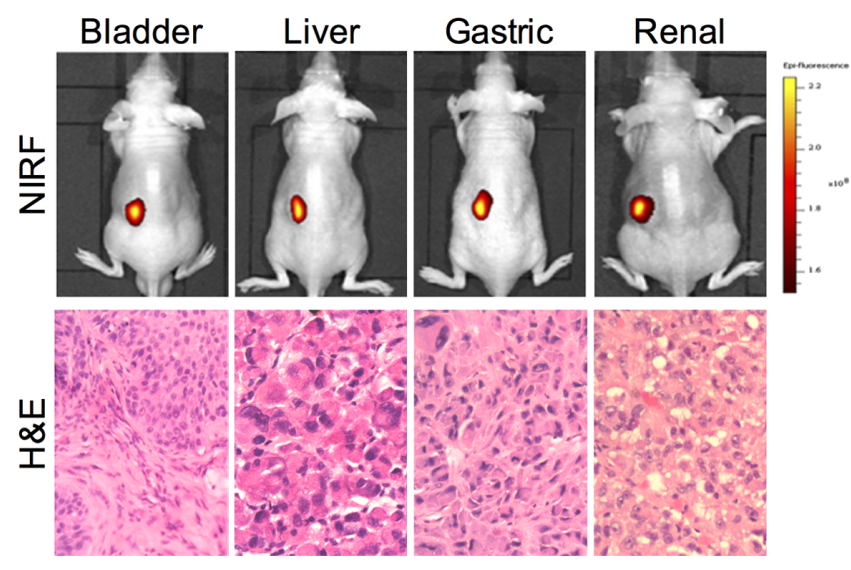

Fig. 2 NIRF imaging of different PDX models with renal capsule xenografts in nude mice. Representative H\&E images indicating tumor histopathology for individual PDX model are shown. Magnification 400x. slight amount of dye retained in normal counterparts. Further quantitative analysis indicated a sixfold increase of MHI-148 dye uptake intensity in tumors relative to normal tissues, with coregistered tumor regions and fluorescence signals confirmed by pathologic analyses. ${ }^{20}$ This pioneering study strongly suggests the potential clinical utility of these attractive NIRF agents.

ICG is the only FDA-approved NIRF agent for clinical use. One of ICG's applications in the clinic is to serve as a preoperative evaluator of liver function by intravenous injection prior to hepatectomy, with the dye being accumulated in hepatocellular carcinoma (HCC) tissue. ${ }^{53}$ The ICG in combination with NIFR imaging of HCC has been developed for intraoperative navigation during open surgery and laparoscopic surgery. ${ }^{54,55}$ But it is known that ICG can make normal tissues emit fluorescence signals with high intensity during some surgical procedures, such as partial nephrectomy, ${ }^{56}$ which is similar to the observations made in ICG-receiving (i.v. route) mouse models for monitoring renal perfusion. ${ }^{57}$ This disadvantage largely compromises the potential use of ICG for tumor visualization but provides opportunities for developing heptamethine cyanine dye-based NIRF imaging approaches in the near future to meet growing clinical needs for better cancer imaging and detection.

\section{Near-Infrared Fluorescence Nuclear Imaging of Cancer}

Nuclear imaging is an attractive modality for cancer detection. ${ }^{30}$ The conventional probes show a short half-life for follow-up use by positron emission computed tomography (PET). Other disadvantages include low spatial resolution, exposure to radiation, and abundant uptake by tissues with high basal metabolic rates, such as the brain. ${ }^{58}$ Since NIRF dyes are able to target cancer cells directly, radioisotope-labeled NIRF dyes are feasible alternative tools for the nuclear imaging of tumors. ${ }^{59}$ NIRF imaging is described within the context of nuclear imaging technologies that remain the "gold standard" of molecular imaging. MR and optical imaging are an attractive combination that can be used to determine both molecular and anatomical information. ${ }^{60}$ NIRMR contrast agents not only provide detailed anatomic imaging but also can be used to image molecular targets at low concentrations with high sensitivity. ${ }^{61}$

\subsection{Tumor Xenograft Mouse Models}

NIRF dyes have been modified by conjugation with a radionuclide and tested for their imaging and targeting potential in xenograft tumor models, showing significant improvements in sensitivity, which suggest the preclinical utility of NIRF dyes for detection of deep-tissue tumors. In a RANKL-overexpressing LNCaP metastatic prostate tumor xenograft model, NIRF imaging detected two superficial tumors in the mouse after PC-1001 injection, while PC-1001-coupled PET revealed an extra tumor in a deep location in the same mouse, ${ }^{30}$ indicating the enhanced sensitivity of NIRF nuclear imaging. To date, several PET tracers have been applied for NIRF nuclear imaging of experimental tumor models, including ${ }^{18} \mathrm{~F},{ }^{11} \mathrm{C},{ }^{99 \mathrm{~m}} \mathrm{Tc},{ }^{64} \mathrm{Cu}$, and ${ }^{111}$ In. ${ }^{31,62-64}$

Recent studies have reported on a PET/NIRF probe, PC- $1001 /{ }^{64} \mathrm{Cu}$, conjugating NIRF dye PC-1001 with ${ }^{64} \mathrm{Cu}$ for breast cancer PET and fluorescence imaging. This $\mathrm{PC}-1001 /{ }^{64} \mathrm{Cu}$ compound has been validated in both breast cancer cells and a breast cancer xenograft model. ${ }^{31}$ It showed 
a much higher rate of uptake and accumulation in tumors compared to normal organs, such as the heart, liver, lung, and spleen, which further exhibited a decrease in the uptake rate with time. However, developing the PET application with this probe in both the research and clinical settings was costly, due to the limited supply of cyclotron-produced ${ }^{64} \mathrm{Cu}$ radioisotope. By contrast, the cancer-targeting SPECT/NIRF dual-modality imaging probe PC-1007/ $/{ }^{99 \mathrm{~m}} \mathrm{Tc}$ has been successfully synthesized in a low-cost scheme and also exhibited cancer-specific targeting and accumulation properties in cancer cells and tumor xenograft models. ${ }^{31}$ The multiple imaging agents were incorporated with Gd(III) chelates and conjugated to IR-783 with a polyethylene glycol (PEG) linker or a short alkyl linker. Both agents achieved outstanding tumor cell labeling capability and were detectable in vivo using both $\mathrm{MR}$ and optical imaging. ${ }^{65}$ Accumulation of these agents in a nude mouse MCF7 tumor xenograft model was detected with fluorescence imaging, and renal clearance by MR imaging. ${ }^{24}$

\subsection{Spontaneous Canine Tumor Models}

To evaluate the tumor-specific targeting ability of NIRF dyes in large live animals, domestic dogs that grew spontaneous tumors were chosen as models to determine the uptake and accumulation of NIRF dyes by both optical imaging and PET/CT scan. ${ }^{22}$ Dogs that carried different spontaneous tumors were injected with MHI-148 dye via femoral vein. After removing the tumors, strong NIR fluorescence signals were detected from primary and metastatic canine tumor tissues, including breast cancer, liver cancer, lung cancer, duodenal cancer, and colon cancer. ${ }^{22}$ It has been demonstrated that these dyes could detect spontaneous tumors in large animals similar to the findings with mice. Since dogs develop spontaneous primary and metastatic tumors in a manner similar to humans, ${ }^{66}$ the specificity and bioavailability of NIRF dyes proven in canine cancer imaging provides new insights into developing NIRF dyes for the clinical detection of human cancers. To further evaluate the tumor-specific targeting ability of NIRF dyes in large live animals, a PC-1001/ ${ }^{68} \mathrm{Ga}$ probe was synthesized by conjugating PC-1001 with ${ }^{68} \mathrm{Ga}$ for independent PET and fluorescence imaging in canine tumor models. PET/CT images clearly displayed the tumor locations by the preferential accumulation of $\mathrm{PC}-1001 /{ }^{68} \mathrm{Ga}$ in the canine tumor tissue versus the normal counterpart. ${ }^{22}$

\subsection{Near-Infrared Fluorescence Imaging-Guided Clinical Surgery}

The application of multimodality optical techniques has been recently brought into focus for better cancer imaging with improved specificity, sensitivity, and reliability as compared to a single modality. Following this trend, bimodal tracers for sentinel lymph node (SLND) by radiolabeled mannosylated dextran derivatives bearing a NIRF imager allowed a clear visualization of the popliteal node by SPECT/CT scan and also enabled the guided identification of SLND during surgery. ${ }^{67}$ In a study of 32 breast cancer patients, the SLNDs of all patients could be identified through radionuclide-combined NIRF imaging in a simple, convenient and high-through putway. ${ }^{68}$ This dual NIRF and radioactive imaging approach further simplifies the early-stage development and validation of in vitro and in vivo optimization of parameters prior to the final PET imaging in live animals and humans.

\section{Near-Infrared Fluorescence Dye-Mediated Targeted Delivery of Antitumor Drugs}

Although a variety of large-molecule delivery systems have been developed in different preclinical and clinical models using peptides, proteins, and polymers, heptamethine cyanine dyes that can serve as small-molecule agents for tumor-specific drug delivery offer several advantages: (1) ease of chemical modification to achieve optimal pharmacological properties, (2) high permeability across cell membranes, and (3) chemical stability and cost effectiveness. ${ }^{32}$

\subsection{Delivery of Conjugated Chemotherapy Drugs}

Theranostic prodrugs equipped with fluorophores as optical reporters have become useful to monitor the drug delivery and release process. Heptamethine cyanine dyes labeled with different antitumor drugs to achieve targeted transport of drugs through the dye-guided specific uptake in tumors allowed both real-time monitoring of therapeutic effect and lower drug dosages to avoid potential side effects (Fig. 3). ${ }^{69}$ Zhang et al. constructed IR-780NM, a tumor-targeting agent by introducing the antitumor drug nitrogen mustard into the parent structure of IR-780 iodide, and demonstrated the utility of this compound for tumor imaging and targeting. ${ }^{28}$ Mostly recently, Wu et al. synthesized the NIR dye-MAOA inhibitor (NMI) compound by conjugating MHI-148 dye with the moiety derived from

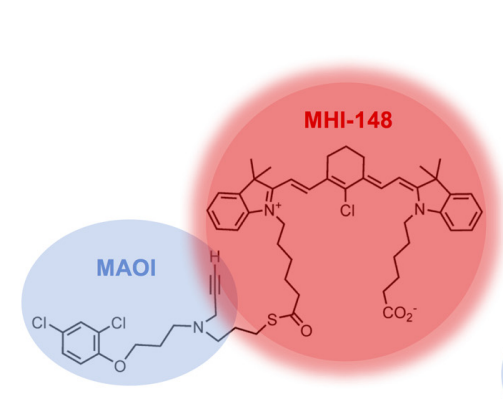

NMI (MHI-148-MAOI)

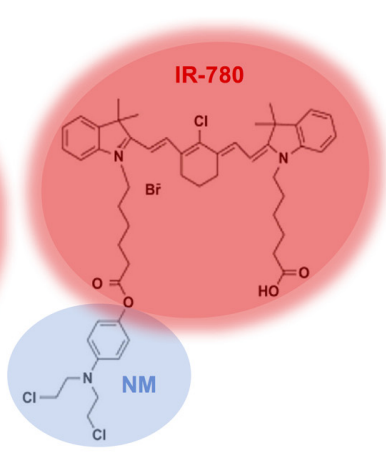

IR-780-NM

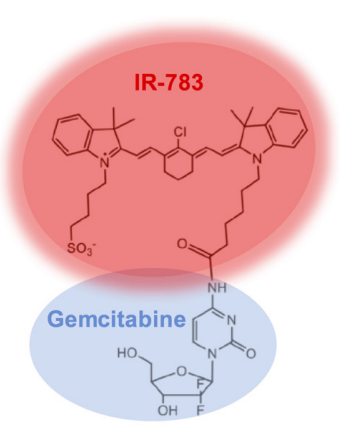

NIRG (IR-783-gemcitabine)

Fig. 3 Chemical structures of NIRF heptamethine cyanine dye-conjugated chemotherapy drugs. The structures of NIR dye and drug in conjugates are shadowed by red and blue colors, respectively. 


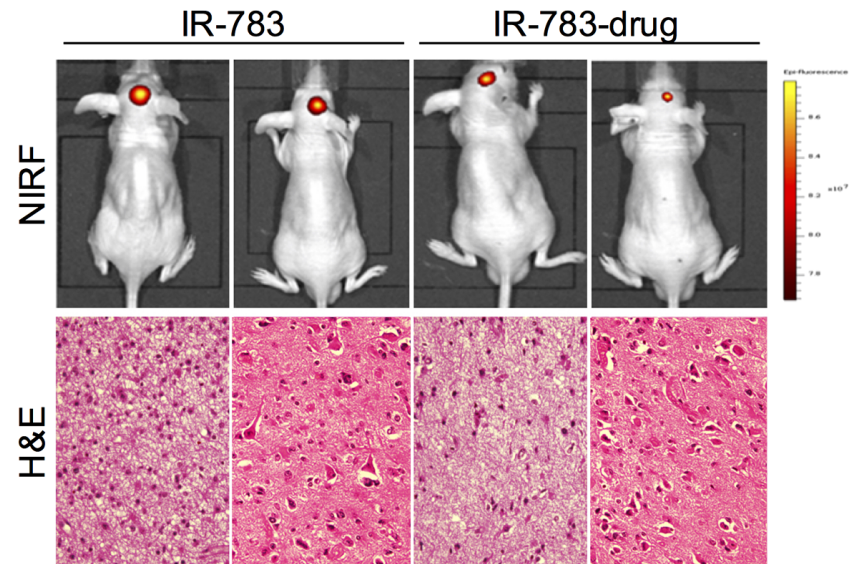

Fig. 4 Preferential uptake and retention of IR-783 dye and derived dye-drug conjugate in intracranial human brain tumor xenograft mouse models. A-B, IR-783 dye; C-D, IR-783-drug conjugate. Representative H\&E images indicating tumor histopathology for individual xenograft are shown. Magnification 400x.

clorgyline, a small molecule inhibitor of monoamine oxidase A that has emerged as a novel therapeutic target for human prostate cancer (Fig. 3). NMI showed tumor-specific targeting properties by systemic circulation in prostate tumor xenograft mice and dramatically restricted tumor growth. This dye-drug conjugate possesses diagnostic potential and can become an important platform for a future generation of anticancer therapeutics. ${ }^{21}$ In addition, mice treated with IR-783-docetaxel conjugate showed significant shrinkage of prostate tumors grown in tibia, with no obvious systemic toxicity or reduced body weight observed in mice during treatment. ${ }^{30}$ These results in aggregate suggest the feasible development of these dyes as a new platform for chemotherapy drug delivery with improved outcomes.

\subsection{Brain Drug Delivery}

Chemotherapy is indispensable for brain tumor treatment after surgery. The major challenge of brain tumor therapy is how to deliver therapeutic agents effectively into the tumor core from the systemic circulation and reduce the side effects of nonspecific biodistribution, which is largely due to poor penetration of drugs through the blood-brain barrier (BBB) and blood-tumor barrier (BTB) ${ }^{70,71}$ After conjugating IR-783 dye to the chemotherapy drug gemcitabine to synthesize a dye-drug conjugate (NIRG) (Fig. 3), we recently showed that the NIRF dye as a drug carrier was able to deliver the drug to brain tumors by penetrating the BBB/BTB in mice (Fig. 4). NIRG treatment also significantly inhibited the growth of intracranial glioma and prostate tumor brain metastases, prolonging survival in mice. ${ }^{25}$ These results demonstrate the great potential of these NIRF dyes and derived dye-drug conjugates for future development and application in the clinic as effective brain tumor theranostics.

\section{Mitochondrial Toxicity}

Mitochondria are one of the major organelles for heptamethine cyanine dye accumulation in cancer cells. ${ }^{27}$ IR-780, a lipophilic molecular agent, has been demonstrated to selectively accumulate in the mitochondria of drug-resistant human lung cancer cells and directly inhibit cancer cell growth, migration and self-renewal capability and delay tumor recurrence. ${ }^{72}$ Mechanistic study revealed that the mitochondrial accumulation of IR-780 increased the production of ROS and depolarization of mitochondrial membrane potential, leading to mitochondrial toxicity and cancer cell apoptosis without the need for any chemical conjugation. ${ }^{29}$ Since the mitochondrial toxicity induced by these dyes was correlated with the increased length of alkyl chain, ${ }^{73}$ Luo et al. designed a series of IR-808 analogs, including IR-808DB, IR-808DH, and IR-808DCH, by increasing the length of the alkyl side chain as well as the lipophilicity of the IR-808 compound, and showed the higher antitumor effects of these analogs, such as IR-808DB, compared to cyclophosphamide in a series of tumor xenograft models. ${ }^{17}$

\section{Photodynamic Therapy}

Several heptamethine cyanine dyes have shown excellent photophysical properties with significant shifts between absorption and emission bands, which could be potentially employed in tumor photodynamic therapy (PDT). ${ }^{74}$ These NIRF dyes selectively aggregate at the tumor site and release cytotoxicity-free radicals to directly kill tumor cells or indirectly inhibit tumor growth by destroying neovascularization. ${ }^{75}$ They can also initiate an inflammatory microenvironment that leads to cancer cell death. ${ }^{76}$ A prototypical heptamethine cyanine compound,

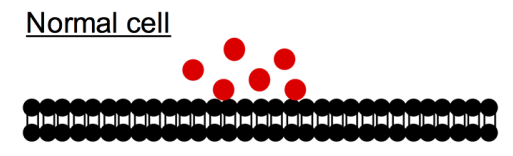

Cytoplasma
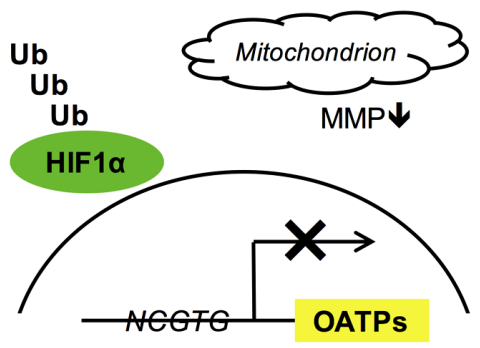

Nucleus
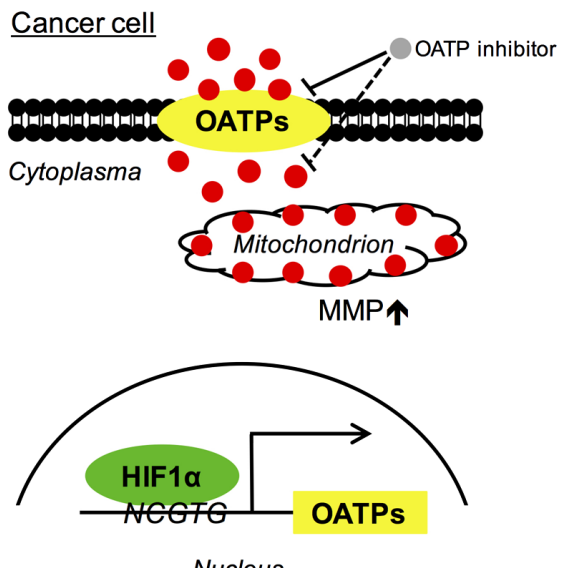

Nucleus

Fig. 5 A schematic outlining the molecular mechanisms underlying the specific uptake of NIRF dyes by cancer cells but not normal cells, which is coordinately mediated by tumor hypoxia, a HIF1 $\alpha /$ OATP signaling axis and mitochondrial membrane potential. 
IR-808, showed photodependent cytotoxic activity. ${ }^{18}$ After receiving NIRF irradiation, it produced light toxicity to tumor cells and inhibited cell proliferation, suggesting its potential role as a theranostic agent for antitumor PDT. ${ }^{17}$ Moreover, IR-808DB, a derivative of IR-808, served as a photosensitizer after structural modification and showed higher sensitivity and efficacy for antitumor PDT.

Improved NIRF dye phototoxicity for PDT applications can also be achieved by structural modification of the metal atoms of the dyes; for instance, the insertion of $\mathrm{Zn}$ in the $\mathrm{Pz} 247$ core was shown to increase the quantum yield of single-oxygen formation. ${ }^{77}$ IR-783 producing photothermal effect leads to thermal ablation of cancer cell. Further loaded with polymeric prodrug micelles exhibited remarkable photothermal cytotoxicity to pancreatic cancer cells ${ }^{78}$ and reversed drug resistance. ${ }^{79}$ The conjugation of IR-783 dye to photosensitizers displays strong potential for NIRF imaging-guided PDT for cancer. ${ }^{38}$ A modified IR-783 after linking with two moieties of the photosensitizer HPPH showed perfect tumor-imaging capability and produced long-term PDT efficacy for mice bearing colon tumors. ${ }^{36}$ Encapsulating NIRF dyes with polymers or inorganic materials to form a variety of nonmetallic nanoparticles also enhanced the photostability, biocompatibility, and fluorescence intensity of dyes with reduced self-aggregation to improve PDT efficacy. ${ }^{42,80-82}$ As a conventional NIR dye, ICG has limited applications as a specific imaging agent for cancer detection because of poor stability and rapid decomposition in polar solution. When ICG was attached to cationic polyethylene to form silicon nanoparticles, the degradation and fluorescence quenching of ICG was largely reduced. ${ }^{83}$ This new ICG-derived product showed good PDT capacity by significantly inhibiting tumor growth in mice after intraperitoneal delivery followed by NIR irradiation. ${ }^{84}$

\section{Mechanisms of Near-Infrared Fluorescence Heptamethine Cyanine Dye Uptake in Cancer Cells}

NIRF heptamethine cyanine dyes are taken up and accumulated by cancer cells through an active transport system, including ATP-driven transport, thus the cellular ability to absorb dyes could be used as a measure for cell viability. ${ }^{85}$ At the molecular level, organic anion-transporting polypeptides (OATPs), a group of cell membrane-bound solute carriers, have been shown to play a key role facilitating the delivery of NIRF dyes into tumor cells. ${ }^{5,19,20,22,28,30}$ This preferential uptake of heptamethine cyanine moiety in tumor is shown to be correlated with enhanced expression of certain type of OATPs in variety of cancer cell types. There are 11 known human OATPs classified into six families and subfamilies on the basis of their amino acid sequence homologies. ${ }^{86}$ OATPs were originally considered to mediate the cellular transport of amphiphilic compounds, including drug and other exogenous materials. ${ }^{87,88}$

\subsection{Tumor Hypoxia and HIF1 $\alpha /$ Organic Anion-Transporting Polypeptides Signaling Axis}

Tumor hypoxia is an important mechanism underlying tumor detection by heptamethine cyanine dye-assisted optical imaging, ${ }^{20,22}$ which is different from ICG that functions primarily through binding to plasma proteins to enable tumor diagnosis by fluorescence imaging. ${ }^{89}$ This is further supported by the lack of evidence indicating a direct association of ICG with tumor hypoxia. In our previous parallel comparison, ICG showed relatively low values of tumor-to-background ratio at 1.4 to 1.7 at $24 \mathrm{~h}$ in tumor-bearing mice by optical imaging, ${ }^{90}$ while MHI-148 had a ratio of 9.1, which could be further increased by twofold in the presence of hypoxia-inducible factor $1 \alpha(\mathrm{HIF} 1 \alpha)$ protein overexpression, a key mediator of hypoxic signaling. ${ }^{20}$ These findings clearly demonstrated the advantage of heptamethine cyanine dyes over other NIRF agents for cancer imaging. Previous studies have demonstrated that OATP1B3, a representative OATP member, dominantly controls the transport of heptamethine cyanine dyes, including IR-780, IR-783, and MHI-148, into cancer cells, which could be antagonized by bromsulphthalein, an OATP competitive inhibitor. ${ }^{19,20,22,23}$ The mechanisms underlying the uptake of NIRF dyes in cancer cells have been shown to be mediated by concerted actions exerted by hypoxia and activation of the HIF $1 \alpha /$ OATPs signaling axis, providing a functional link between tumor hypoxia and OATPs to enhance dye uptake. ${ }^{20,22}$ As shown in Fig. 5, tumor hypoxia, a frequent phenomenon in a wide range of cancers and typically associated with changes in metabolism, neovascularization, invasion, metastasis, drug resistance, and ultimately poor clinical outcomes, ${ }^{91,92}$ mediates dye uptake mainly through HIF1 $\alpha$, which is capable of directly upregulating OATPs. ${ }^{20}$ Under hypoxic conditions, active nuclear HIF1 $\alpha$ protein interacts with a hypoxia response element (HRE) in the OATP promoter, resulting in increased transcription of OATP genes. By contrast, HIF1 $\alpha$ is destabilized under normoxia due to rapid degradation initiated by the PHD/VHL pathway, ${ }^{29}$ which is likely the mechanism responsible for the relatively low levels of OATPs generally observed in normal tissues. ${ }^{93}$ Thus, inactivation of HIF $1 \alpha /$ OATPs signaling in normal cells and tissues blunts their sensitivity to recruit NIRF dyes for the long-lasting retention and preferential organelle-specific cellular localization seen in cancer cells. The proposed regulatory relationship between hypoxia/HIF1 $\alpha$ and OATPs was supported by observations that treatment of cancer cells with HIF $1 \alpha$ stabilizers, such as cobalt chloride and DMOG, induced the transcription levels of select isoforms of OATPs in addition to known HIF1 $\alpha$-target genes, which was correlated with increased uptake of NIRF dye by cancer cells. Alternatively, genetic knockdown of HIF $1 \alpha$ in cancer cells reduced the expression of select OATPs, such as OATP1B3 and OATP2B1, along with HIF $1 \alpha$-responsive genes, corresponding with reduced uptake of NIRF dye by cancer cells. ${ }^{20,22}$ These mechanisms have been well demonstrated in prostate cancer and brain cancer so far, ${ }^{20,22,25}$ and are expected to work in other cancers as well given the prevailing activation of tumor hypoxia and OATP expression in most types of cancers. ${ }^{94,95}$ In addition, we have recently established a NIRF imaging method to analyze clinical tumor samples ex vivo, where the activation of HIF1 $\alpha /$ OATPs signaling could be recapitulated, evidenced by intense widespread nuclear HIF1 $\alpha$ and cytoplasmic OATP1B3 protein expression in RCC samples but not adjacent normal tissues. ${ }^{20}$

\subsection{Enhanced Permeability and Retention Effect}

In addition to the well-elucidated hypoxia/HIF1 $\alpha$ - and OATPsmediated mechanisms, alternative mechanisms may also be used by some NIRF dyes, such as IR-780 and Pz 247. ${ }^{28,96}$ The uptake of IR-780 iodide by tumor cells has been shown to be dependent on cellular energy metabolism, plasma/mitochondrial membrane 
potential, and membrane-bound OATPs. ${ }^{28}$ Both OATP1B3, an influx transporter and multidrug resistance p-glycoprotein-3 (MDR3), an efflux transporter, as main ICG-related transporters play pivotal roles in the uptake of ICG in HCC. The uptake of ICG mediated by OATP1B3 and impaired biliary excretion of ICG caused by MDR3 contribute to the accumulation of ICG in HCC tissues. ${ }^{53}$ In addition, Ishizawa et al. found that the portal uptake of ICG in HCC cells is mediated by $\mathrm{Na}^{+} /$taurocholate transport protein and OATP1B3 after preoperative intravenous administration. $^{54}$

Serum components, such as albumin and low-density lipoprotein (LDL), play important roles in the delivery of hydrophobic NIRF dye to cells. For example, $\mathrm{Pz} 247$ is taken up by cells into lysosomes through LDL receptor-mediated endocytosis, leading to accumulation in lysosomes and accompanied NIR emission. ${ }^{77}$ Addition of heparinto at a dose of $25 \mathrm{mg} / \mathrm{mL}$ inhibited LDL binding to LDL receptor and completely eliminated the lysosomal localization of $\mathrm{Pz} 247$. Along with the elevated LDL-bound cholesterol in highly proliferative tumor cells relative to normal cells, $\mathrm{Pz} 247$ has been suggested for a potential application for targeting tumor cells. ${ }^{96}$ In short, heptamethine cyanine dyes can achieve organelle-preferential accumulation in cancer cells by enhanced permeability and retention (EPR) effect, which facilitates the entrance of dyes to the intracellular compartment with higher retention in the mitochondria and lysosomes through transmembrane transport proteins or endocytosis. ${ }^{19,77}$

\subsection{Reactive Sulfur Species}

The targeting of mitochondria by NIRF dyes may be closely related to intracellular reactive sulfur species. Endogenous hydrogen sulfide is an important gasotransmitter involved in critical physiological and pathological processes. ${ }^{97}$ A NIRF multiresponse probe, $\mathrm{Cy}-\mathrm{NO} 2$, was designed for hydrogen sulfide detection and imaging in live cells. ${ }^{98}$ Further study found that sulfane sulfur instead of $\mathrm{H}_{2} \mathrm{~S}$ is the actual signaling molecule, which can be generated as a result of the reaction between $\mathrm{O}_{2}^{-}$and $\mathrm{H}_{2} \mathrm{~S}$ in the mitochondria. ${ }^{99} \mathrm{~A}$ dual response NIRF probe was employed to image $\mathrm{O}_{2}^{-} / \mathrm{H}_{2} \mathrm{~S}$ in cells and in vivo. This probe was derived from the NIRF heptamethine cyanine dye Cy.7. $\mathrm{Cl}{ }^{100}$ Because mitochondria are the main production source of ROS in cells, and the mitochondrial fraction contains $\sim 60 \%$ of bound sulfane sulfur, ${ }^{101}$ this probe specifically targets and localizes in mitochondria and can be employed to directly detect changes in mitochondrial $\mathrm{O}_{2}^{-}$and $\mathrm{H}_{2} \mathrm{~S} .{ }^{99}$

\section{Conclusions and Future Perspectives}

Although significant recent progress has been made in developing heptamethine cyanine dye-based NIRF imaging methods, further improvements of these dyes through a series of critical preclinical steps are needed prior to their ultimate clinical use.

\subsection{Chemical Modification}

Additional chemical conjugations may be needed to enhance the EPR ability of currently available heptamethine cyanine dyes to adjust their lipid and water solubility, to facilitate their transportation through cell membranes and effective recognition of intracellular molecules, to weaken background interference by lowering noncovalent hydrophobic binding of probes to proteins in blood, and to reduce toxicity to normal cells. ${ }^{102}$ Moreover, a series of synthetic modifications should be addressed, such as solubility improvement to allow higher injection doses or an alternate route of administration. In addition, given that heptamethine cyanine dyes show preferential accumulation and retention mainly in the mitochondria, which contain most of the sulfane sulfur, we will also be able to design novel NIRF probes for hydrogen polysulfides to further improve mitochondrial targeting. ${ }^{7}$

The ideal fluorescence probe should display high sensitivity in living cells. Improvement of intracellular retention of NIRF dye is a new approach to increase the sensitivity of fluorescence. ${ }^{103}$ Given that individual OATP transport substrates with compound specificity, a highly sensitive, high-throughput fluorescencebased OATP1B1 inhibition assay system was established to screen the most sensitive fluorescence. ${ }^{104}$ Several fluorescein derivatives as substrates of OATPs were evaluated for their uptake via OATP1B1-expressing human HEK293 cells. ${ }^{105}$ By determination of substrate-dependent $K_{i}$ variations, dichlorofluorescein showed the highest OATP1B1-mediated uptake among all fluorescent compounds examined. In line with the maintenance of OATP substrate specificity, several structure optimizations of NIRF heptamethine cyanine dyes can be further made by detection of substrate-dependent $K_{i}$ value.

Protein nanoparticles have been reported to protect NIRF dyes from being destroyed by ROS and reduce bleaching, thereby enhancing the fluorescence stability of the dyes. ${ }^{106}$ Protein nanoparticles are more compatible than other forms of nanoparticles and thus can be used as transport carriers for a number of chemotherapy drugs in tumor-targeted therapy. For example, the NIR prodrug dicyanomethylene-disulfidecamptothecin and its derivative, PEG-polylactic acid, when loaded in nanoparticles, emitted NIRF signals. ${ }^{107,108}$ These NIR-encapsulated nanoparticles showed higher antitumor activity and longer retention in the plasma than free camptothecin. ${ }^{108}$ Additionally, the excellent fluorescence intensity and photostability of DCM chromophores with a large Stokes shift makes the in vivo and in situ tracking of drug release and therapeutic efficacy in live animals possible. ${ }^{108}$

\subsection{Preclinical Experiments}

The penetrability and sensitivity of NIRF dyes need to be improved before clinical use. For example, NIRF dyes can be labeled with radionuclide for combined PET/CT scanning for early diagnosis of microtumors in vivo. ${ }^{32,109}$ Bright NIRF agents further conjugated to disease-targeting moieties promise molecular imaging and image-guided surgery. In addition to the mouse or rat tumor models usually preferred for preclinical testing, it would be worthwhile to verify the reliability and sensibility of NIRF imaging in large animal models, such as dog or pig models carrying different types of spontaneous tumors, to address essential issues in human cancers that are not reflected in small animal model systems. ${ }^{110}$ Finally, crucial evaluations of pharmacokinetics, pharmacodynamics, and toxicity of dyes and dye-drug conjugates are also needed in mouse and dog models before this group of novel compounds can be moved into the clinic for improved cancer diagnosis, prognosis and therapy. ${ }^{108}$ Taking into account the complexity of targeted drug development, the development of diagnostic NIRF agents for tumor imaging should give the priority to the selection conducted in preclinical experimental models, where both the PDX mouse model and dog spontaneous tumor model are obviously convenient tools for preclinical tests.

In summary, a group of NIRF heptamethine cyanine dyes with dual imaging and targeting properties are promising 
novel anticancer theranostic agents. These NIRF dyes and their derivatives can be exploited for the detection and treatment of different types of tumors to meet preclinical and clinical needs without chemical conjugation. Although obstacles remain, valuable and exciting opportunities exist in this field.

\section{Acknowledgments}

This work was supported by Laboratory Animal Science Funding Program under Grant No. SYDW2014-002 and National Natural Science Foundation Program under Grant No. 31572340. We thank Gary Mawyer for editorial assistance. The authors declare no potential conflicts of interest with respect to the authorship and/or publication of this article.

\section{References}

1. C. S. Yeh et al., "Tumor targeting and MR imaging with lipophilic cyanine-mediated near-infrared responsive porous $\mathrm{Gd}$ silicate nanoparticles," Biomaterials 34(22), 5677-5688 (2013).

2. J. V. Frangioni, "In vivo near-infrared fluorescence imaging," Curr. Opin. Chem. Biol. 7(5), 626-634 (2003).

3. A. J. Mieszawska et al., "Synthesis of polymer-lipid nanoparticles for image-guided delivery of dual modality therapy," Bioconjugate Chem. 24(9), 1429-1434 (2013).

4. A. Papadia et al., "Indocyanine green fluorescence imaging in the surgical management of an iatrogenic lymphatic fistula: description of a surgical technique," J. Minimally Invasive Gynecol. 22(7), 13041306 (2015).

5. X. Yi et al., "IR-780 dye for near-infrared fluorescence imaging in prostate cancer," Med. Sci. Monit. 21, 511-517 (2015).

6. C. Shao et al., "Detection of live circulating tumor cells by a class of near-infrared heptamethine carbocyanine dyes in patients with localized and metastatic prostate cancer," PloS One 9(2), e88967 (2014).

7. G. Liberale et al., "Fluorescence imaging after intraoperative intravenous injection of indocyanine green for detection of lymph node metastases in colorectal cancer," Eur. J. Surg. Oncol. 41(9), 12561260 (2015).

8. A. K. Kirchherr, A. Briel, and K. Mader, "Stabilization of indocyanine green by encapsulation within micellar systems," Mol. Pharmaceutics 6(2), 480-491 (2009).

9. O. A. Andreev et al., "Mechanism and uses of a membrane peptide that targets tumors and other acidic tissues in vivo," Proc. National Acad. Sci. U. S. A. 104(19), 7893-7898 (2007).

10. J. A. Yang et al., "Hyaluronic acid-tumor necrosis factor-related apoptosis-inducing ligand conjugate for targeted treatment of liver fibrosis," Acta Biomater. 12, 174-182 (2015).

11. S. Keereweer et al., "Targeting integrins and enhanced permeability and retention (EPR) effect for optical imaging of oral cancer," J. Surg. Oncol. 105(7), 714-718 (2012).

12. M. Xu et al., "Preclinical evaluation of Mab CC188 for ovarian cancer imaging," Int. J. Cancer 131(6), 1351-1359 (2012).

13. J. Gao et al., "Affibody-based nanoprobes for HER2-expressing cell and tumor imaging," Biomaterials 32(8), 2141-2148 (2011).

14. S. Luo et al., "A review of NIR dyes in cancer targeting and imaging," Biomaterials 32(29), 7127-7138 (2011).

15. X. Yi et al., "Near-infrared fluorescent probes in cancer imaging and therapy: an emerging field," Int. J. Nanomed. 9, 1347-1365 (2014).

16. X. Yang et al., "Optical imaging of kidney cancer with novel near infrared heptamethine carbocyanine fluorescent dyes," J. Urol. 189(2), 702710 (2013).

17. S. Luo et al., "A multifunctional heptamethine near-infrared dye for cancer theranosis," Biomaterials 34(9), 2244-2251 (2013).

18. X. Tan et al., "A NIR heptamethine dye with intrinsic cancer targeting, imaging and photosensitizing properties," Biomaterials 33(7), 22302239 (2012).

19. X. Yang et al., "Near IR heptamethine cyanine dye-mediated cancer imaging," Clin. Cancer Res. 16(10), 2833-2844 (2010).

20. J. B. Wu et al., "Near-infrared fluorescence imaging of cancer mediated by tumor hypoxia and HIF1alpha/OATPs signaling axis," Biomaterials 35(28), 8175-8185 (2014).
21. J. B. Wu et al., "Monoamine oxidase a inhibitor-near-infrared dye conjugate reduces prostate tumor growth," J. Am. Chem. Soc. 137(6), 23662374 (2015).

22. C. Shi et al., "Heptamethine carbocyanine dye-mediated near-infrared imaging of canine and human cancers through the HIF-1alpha/OATPs signaling axis," Oncotarget 5(20), 10114-10126 (2014).

23. J. Yuan et al., "Nearinfrared fluorescence imaging of prostate cancer using heptamethine carbocyanine dyes," Mol. Med. Rep. 11(2), 821828 (2015).

24. V. S. Harrison et al., "Multimeric near IR-MR contrast agent for multimodal in vivo imaging," J. Am. Chem. Soc. 137(28), 9108-9116 (2015).

25. J. B. Wu et al., "Near-infrared fluorescence heptamethine carbocyanine dyes mediate imaging and targeted drug delivery for human brain tumor," Biomaterials 67, 1-10 (2015).

26. A. Fernandez-Fernandez et al., "Comparative study of the optical and heat generation properties of IR820 and indocyanine green," Mol. Imaging 11(2), 99-113 (2012).

27. C. Zhang et al., "A near-infrared fluorescent heptamethine indocyanine dye with preferential tumor accumulation for in vivo imaging," Biomaterials 31(25), 6612-6617 (2010).

28. E. Zhang et al., "Mechanistic study of IR-780 dye as a potential tumor targeting and drug delivery agent," Biomaterials 35(2), 771-778 (2014).

29. Y. Wang et al., "Preferential accumulation of the near infrared heptamethine dye IR-780 in the mitochondria of drug-resistant lung cancer cells," Biomaterials 35(13), 4116-4124 (2014).

30. J. Wu, D. Pan, and L. W. Chung, "Near-infrared fluorescence and nuclear imaging and targeting of prostate cancer," Transl. Androl. Urol. 2(3), 254-264 (2013).

31. Y. Zhang et al., "Novel cancer-targeting SPECT/NIRF dual-modality imaging probe (99m)Tc-PC-1007: synthesis and biological evaluation," Bio-org. Med. Chem. Lett. 23(23), 6350-6354 (2013).

32. L. Xiao et al., "Heptamethine cyanine based (64)Cu-PET probe PC1001 for cancer imaging: synthesis and in vivo evaluation," Nucl. Med. Biol. 40(3), 351-360 (2013).

33. C. Li et al., "Synthesis and characterization of glucosamine-bound nearinfrared probes for optical imaging," Org. Lett. 8(17), 3623-3626 (2006).

34. D. S. Conceicao, D. P. Ferreira, and L. F. Ferreira, "Photochemistry and cytotoxicity evaluation of heptamethinecyanine near infrared (NIR) dyes," Int. J. Mol. Sci. 14(9), 18557-18571 (2013).

35. K. Licha et al., "Hydrophilic cyanine dyes as contrast agents for nearinfrared tumor imaging: synthesis, photophysical properties and spectroscopic in vivo characterization," Photochem. Photobiol. 72(3), 392-398 (2000).

36. N. S. James et al., "Comparative tumor imaging and PDT Efficacy of HPPH conjugated in the mono- and di-forms to various polymethine cyanine dyes: part-2," Theranostics 3(9), 703-718 (2013).

37. B. R. Renikuntla et al., "Improved photostability and fluorescence properties through polyfluorination of a cyanine dye," Org. Lett. 6(6), 909912 (2004).

38. N. S. James et al., "Evaluation of polymethine dyes as potential probes for near infrared fluorescence imaging of tumors: part-1," Theranostics 3(9), 692-702 (2013).

39. L. Yuan et al., "Far-red to near infrared analyte-responsive fluorescent probes based on organic fluorophore platforms for fluorescence imaging," Chem. Soc. Rev. 42(2), 622-661 (2013).

40. H. Lee, J. C. Mason, and S. Achilefu, "Synthesis and spectral properties of near-infrared aminophenyl-, hydroxyphenyl-, and phenyl-substituted heptamethine cyanines," J. Org. Chem. 73(2), 723-725 (2008).

41. C. Zhang et al., "Sentinel lymph node mapping by a near-infrared fluorescent heptamethine dye," Biomaterials 31(7), 1911-1917 (2010).

42. C. Yue et al., "IR-780 dye loaded tumor targeting theranostic nanoparticles for NIR imaging and photothermal therapy," Biomaterials 34(28), 6853-6861 (2013).

43. E. Engel et al., "Light-induced decomposition of indocyanine green," Invest. Ophthalmol. Visual Sci. 49(5), 1777-1783 (2008).

44. G. T. Spence et al., "Near-infrared croconaine rotaxanes and doped nanoparticles for enhanced aqueous photothermal heating," Chemistry 20(39), 12628-12635 (2014).

45. S. Guha et al., "Clean photothermal heating and controlled release from near-infrared dye doped nanoparticles without oxygen photosensitization," Langmuir 31(28), 7826-7834 (2015). 
46. E. R. Trivedi et al., "Chiral bis-acetal porphyrazines as near-infrared optical agents for detection and treatment of cancer," Photochem. Photobiol. 86(2), 410-417 (2010).

47. Y. Tian et al., "A rapid and convenient method for detecting a broad spectrum of malignant cells from malignant pleuroperitoneal effusion of patients using a multifunctional NIR heptamethine dye," Analyst 140(3), 750-755 (2015).

48. D. Siolas and G. J. Hannon, "Patient-derived tumor xenografts: transforming clinical samples into mouse models," Cancer Res. 73(17), 5315-5319 (2013).

49. J. L. Green et al., "Paracrine Wnt signaling both promotes and inhibits human breast tumor growth," Proc. Natl. Acad. Sci. U. S. A. 110(17), 6991-6996 (2013).

50. H. Xin et al., "Establishment and characterization of 7 novel hepatocellular carcinoma cell lines from patient-derived tumor xenografts," PloS One 9(1), e85308 (2014).

51. T. K. Hill et al., "Indocyanine green-loaded nanoparticles for imageguided tumor surgery," Bioconjugate Chem. 26(2), 294-303 (2015).

52. J. R. van der Vorst et al., "Near-infrared fluorescence sentinel lymph node mapping of the oral cavity in head and neck cancer patients," Oral Oncol. 49(1), 15-19 (2013).

53. Y. Shibasaki et al., "Expression of indocyanine green-related transporters in hepatocellular carcinoma," J. Surg. Res. 193(2), 567-576 (2015).

54. T. Ishizawa et al., "Mechanistic background and clinical applications of indocyanine green fluorescence imaging of hepatocellular carcinoma," Ann. Surg. Oncol. 21(2), 440-448 (2014).

55. T. Ishizawa et al., "Real-time identification of liver cancers by using indocyanine green fluorescent imaging," Cancer 115(11), 2491-2504 (2009).

56. M. A. Bjurlin, T. R. McClintock, and M. D. Stifelman, "Near-infrared fluorescence imaging with intraoperative administration of indocyanine green for robotic partial nephrectomy," Curr. Urol. Rep. 16(4), 20 (2015).

57. N. Arichi et al., "Intraoperative fluorescence vascular imaging using indocyanine green for assessment of transplanted kidney perfusion," Transplant. Proc. 46(2), 342-345 (2014).

58. M. A. Pysz, S. S. Gambhir, and J. K. Willmann, "Molecular imaging: current status and emerging strategies," Clin. Radiol. 65(7), 500-516 (2010).

59. J. S. Kim et al., "Development and in vivo imaging of a PET/MRI nanoprobe with enhanced NIR fluorescence by dye encapsulation," Nanomedicine 7(2), 219-229 (2012).

60. P. Verwilst et al., "Recent advances in Gd-chelate based bimodal optical/ MRI contrast agents," Chem. Soc. Rev. 44(7), 1791-1806 (2015).

61. E. M. Sevick-Muraca, "Translation of near-infrared fluorescence imaging technologies: emerging clinical applications," Ann. Rev. Med. 63, 217-231 (2012).

62. A. Azhdarinia et al., "Dual-labeling strategies for nuclear and fluorescence molecular imaging: a review and analysis," Mol. Imaging Boil. 14(3), 261-276 (2012).

63. Y. Hong et al., "Synthesis and radiolabeling of (111)In-core-cross linked polymeric micelle-octreotide for near-infrared fluoroscopy and single photon emission computed tomography imaging," Bio-org. Med. Chem. Lett. 24(12), 2781-2785 (2014).

64. S. Lutje et al., "Pretargeted dual-modality immuno-SPECT and nearinfrared fluorescence imaging for image-guided surgery of prostate cancer," Cancer Res. 74(21), 6216-6223 (2014).

65. V. S. Harrison et al., "A multimeric MR-optical contrast agent for multimodal imaging," Chem. Commun. 50(78), 11469-11471 (2014).

66. B. E. Leroy and N. Northrup, "Prostate cancer in dogs: comparative and clinical aspects," Vet. J. 180(2), 149-162 (2009).

67. J. R. van der Vorst et al., "Dose optimization for near-infrared fluorescence sentinel lymph node mapping in patients with melanoma," $B r . J$. Dermatol. 168(1), 93-98 (2013).

68. B. E. Schaafsma et al., "Clinical trial of combined radio- and fluorescence-guided sentinel lymph node biopsy in breast cancer," Br. J. Surg. 100(8), 1037-1044 (2013).

69. Y. Jiao et al., "Tumor-targeting multifunctional rattle-type theranostic nanoparticles for MRI/NIRF bimodal imaging and delivery of hydrophobic drugs," Small 11(16), 1962-1974 (2015).

70. S. Agarwal et al., "Function of the blood-brain barrier and restriction of drug delivery to invasive glioma cells: findings in an orthotopic rat xenograft model of glioma," Drug Metabol. Dispos. 41(1), 33-39 (2013).
71. D. R. Groothuis, "The blood-brain and blood-tumor barriers: a review of strategies for increasing drug delivery," Neuro-Oncology 2(1), 45-59 (2000).

72. Y. Suo et al., "Near infrared in vivo flow cytometry for tracking fluorescent circulating cells," Cytometry A 87(9), 878-884 (2015).

73. M. Krieg and J. M. Bilitz, "Structurally modified trimethine thiacarbocyanine dyes. Effect of $\mathrm{N}$-alkyl substituents on antineoplastic behavior," Biochem. Pharmacol. 51(11), 1461-1467 (1996).

74. R. Radzi et al., "Photodynamic hyperthermal therapy with indocyanine green (ICG) induces apoptosis and cell cycle arrest in B16F10 murine melanoma cells," J. Vet. Med. Sci./Jpn. Soc. Vet. Sci. 74(5), 545-551 (2012).

75. G. Shafirstein et al., "Indocyanine green enhanced near-infrared laser treatment of murine mammary carcinoma," Int. J. Cancer 130(5), 1208-1215 (2012).

76. A. M. Gamal-Eldeen et al., "Photodynamic therapeutic role of indocyanine green in tumor-associated inflammation in skin cancer," Photodiagn. Photodyn. Ther. 11(2), 239-249 (2014).

77. E. R. Trivedi et al., "Chiral porphyrazine near-IR optical imaging agent exhibiting preferential tumor accumulation," Proc. Natl. Acad. Sci. U. S. A. 107(4), 1284-1288 (2010).

78. Y. Chen et al., "IR-780 loaded phospholipid mimicking homopolymeric micelles for near-IR imaging and photothermal therapy of pancreatic cancer," ACS Appl. Mater. Interfaces 8(11), 6852-6858 (2016).

79. Z. Li et al., "pH- and NIR light-responsive polymeric prodrug micelles for hyperthermia-assisted site-specific chemotherapy to reverse drug resistance in cancer treatment," Small (2016) (Epub ahead of print).

80. M. Kolitz-Domb et al., "Engineering of near infrared fluorescent proteinoid-poly(L-lactic acid) particles for in vivo colon cancer detection," J. Nanobiotechnol. 12, 30 (2014).

81. R. H. Patel et al., "Multifunctionality of indocyanine green-loaded biodegradable nanoparticles for enhanced optical imaging and hyperthermia intervention of cancer," J. Biomed. Opt. 17(4), 046003 (2012).

82. G. Shan, R. Weissleder, and S. A. Hilderbrand, "Upconverting organic dye doped core-shell nano-composites for dual-modality NIR imaging and photo-thermal therapy," Theranostics 3(4), 267-274 (2013).

83. B. Quan et al., "Near infrared dye indocyanine green doped silica nanoparticles for biological imaging," Talanta 99, 387-393 (2012).

84. X. Zheng et al., "Indocyanine green-containing nanostructure as near infrared dual-functional targeting probes for optical imaging and photothermal therapy," Mol. Pharmaceutics 8(2), 447-456 (2011).

85. J. C. Rasmussen et al., "Human lymphatic architecture and dynamic transport imaged using near-infrared fluorescence," Transl. Oncol. 3(6), 362-372 (2010).

86. B. Hagenbuch and B. Stieger, "The SLCO (former SLC21) superfamily of transporters," Mol. Aspects Med. 34(2-3), 396-412 (2013).

87. T. Liu and Q. Li, "Organic anion-transporting polypeptides: a novel approach for cancer therapy," J. Drug Target. 22(1), 14-22 (2014).

88. I. Tamai and T. Nakanishi, "OATP transporter-mediated drug absorption and interaction," Curr. Opin. Pharmacol. 13(6), 859-863 (2013).

89. C. Hirche et al., "ICG fluorescence-guided sentinel node biopsy for axillary nodal staging in breast cancer," Breast Cancer Res. Treat. 121(2), 373-378 (2010).

90. N. Kosaka et al., "Near infrared fluorescence-guided real-time endoscopic detection of peritoneal ovarian cancer nodules using intravenously injected indocyanine green," Int. J. Cancer 129(7), 16711677 (2011)

91. D. M. Gilkes and G. L. Semenza, "Role of hypoxia-inducible factors in breast cancer metastasis," Future Oncol. 9(11), 1623-1636 (2013).

92. J. Liao et al., "Ovarian cancer spheroid cells with stem cell-like properties contribute to tumor generation, metastasis and chemotherapy resistance through hypoxia-resistant metabolism," PloS One 9(1), e84941 (2014).

93. A. Obaidat, M. Roth, and B. Hagenbuch, "The expression and function of organic anion transporting polypeptides in normal tissues and in cancer," Ann. Rev. Pharmacol. Toxicol. 52, 135-151 (2012).

94. S. Han et al., "Role of hypoxia inducible factor-1alpha in the regulation of the cancer-specific variant of organic anion transporting polypeptide 1B3 (OATP1B3), in colon and pancreatic cancer," Biochem. Pharmacol. 86(6), 816-823 (2013).

95. Z. Hu et al., "A compact VEGF signature associated with distant metastases and poor outcomes," BMC Med. 7, 9 (2009). 
96. S. Lee et al., "Tuning the singlet oxygen quantum yield of near-IRabsorbing porphyrazines," Photochem. Photobiol. 77(1), 18-21 (2003).

97. B. D. Paul and S. H. Snyder, "H(2)S signalling through protein sulfhydration and beyond," Nat. Rev. Mol. Cell Biol. 13(8), 499-507 (2012).

98. R. Wang et al., "A highly selective turn-on near-infrared fluorescent probe for hydrogen sulfide detection and imaging in living cells," Chem. Commun. 48(96), 11757-11759 (2012).

99. F. Yu et al., "A dual response near-infrared fluorescent probe for hydrogen polysulfides and superoxide anion detection in cells and in vivo," Biomaterials 63, 93-101 (2015).

100. K. Kundu et al., "Hydrocyanines: a class of fluorescent sensors that can image reactive oxygen species in cell culture, tissue, and in vivo," Angew. Chem. 48(2), 299-303 (2009).

101. N. Shibuya et al., "3-Mercaptopyruvate sulfurtransferase produces hydrogen sulfide and bound sulfane sulfur in the brain," Antioxid. Redox Signal. 11(4), 703-714 (2009).

102. T. Liu et al., "Synthesis and characterization of a glycine-modified heptamethine indocyanine dye for in vivo cancer-targeted near-infrared imaging," Drug Des. Dev. Ther. 8, 1287-1297 (2014).

103. S. Izumi et al., "A simple and effective strategy to increase the sensitivity of fluorescence probes in living cells," J. Am. Chem. Soc. 131(29), 10189-10200 (2009).

104. M. Roth, A. Obaidat, and B. Hagenbuch, "OATPs, OATs and OCTs: the organic anion and cation transporters of the SLCO and SLC22A gene superfamilies," Br. J. Pharmacol. 165(5), 1260-1287 (2012).
105. S. Izumi et al., "Investigation of fluorescein derivatives as substrates of organic anion transporting polypeptide (OATP) 1B1 to develop sensitive fluorescence-based OATP1B1 inhibition assays," Mol. Pharmaceutics (2016) (Epub ahead of print).

106. S. Cohen and S. Margel, "Engineering of near IR fluorescent albumin nanoparticles for in vivo detection of colon cancer," J. Nanobiotechnol. 10, 36 (2012).

107. A. Schadlich et al., "Tumor accumulation of NIR fluorescent PEGPLA nanoparticles: impact of particle size and human xenograft tumor model," ACS Nano 5(11), 8710-8720 (2011).

108. X. Wu et al., "In vivo and in situ tracking cancer chemotherapy by highly photostable NIR fluorescent theranostic prodrug," J. Am. Chem. Soc. 136(9), 3579-3588 (2014).

109. C. Perez-Medina et al., "A modular labeling strategy for in vivo PET and near-infrared fluorescence imaging of nanoparticle tumor targeting," J. Nucl. Med. 55(10), 1706-1711 (2014).

110. M. Paoloni and C. Khanna, "Translation of new cancer treatments from pet dogs to humans," Nat. Rev. Cancer 8(2), 147-156 (2008).

Changhong Shi is professor of Laboratory Animal Science at the Fourth Military Medical University. He has a PhD in tumor pathology, and his research is in the optical imaging of tumor animal model. He has established many different types of patient-derived tumor xenograft (PDX) model to evaluate heptamethine carbocyanine dye. The relative work has been published in a number of different journals including Oncotarget, Biomaterials and Toxicology.

Biographies for the other authors are not available. 